\title{
Saltwater Upcoming and Decay Beneath a Well Pumping Above an Interface Zone
}

\author{
Quanlin Zhou ${ }^{a}$, Jacob Bear ${ }^{b}$, and Jacob Bensabat ${ }^{c}$ \\ ${ }^{\text {a }}$ Earth Sciences Division, Lawrence Berkeley National Laboratory, Berkeley, CA 94720, USA \\ ${ }^{\mathbf{b}}$ Faculty of Civil \& Environmental Eng, Technion-Israel Inst. of Technology, Haifa, 32000, Israel \\ ${ }^{\mathbf{c}}$ Environmental and Water Resources Engineering Inc., PO Box 6770, Haifa, 31067, Israel
}

\begin{abstract}
Saltwater, or brine, underlies fresh water in many aquifers, with a transition zone separating them. Pumping fresh water by wells located above the transition zone produces upconing of the latter, eventually salinizing the pumped water, forcing shut-off. The salinity of the pumped water depends on the pumping rate, on the location of the well's screen, on the fresh water flow regime, and on the difference in density between fresh and salt water, expressed as a dimensionless factor called density difference factor (DDF). Following the well's shut-off, the upconed saltwater mound undergoes decay, tending to return to the prepumping regime. In this paper, the upconing-decay processes in an axially symmetrical system are investigated to discover how they are affected by the DDF and by the dispersivities. The code FEAS-Brine, developed for the simulation of coupled density-dependent flow and salt transport, is used. In this code, the flow equation is solved by the Galerkin finite element method (FEM), while the advective-dispersive salt transport equation is solved in the EulerianLagrangian framework. This code does not suffer from the instability constraint on the Peclet number in the vicinity of the pumping well, where advection dominates the salt transport. Simulation results show that upconing is very sensitive to the DDF, which, in our work, is in the range from 0 (for ideal tracer) to 0.2 (for brine). It is shown that for the DDF of 0.025 (for seawater), local upconing occurs only for low iso-salinity surfaces, while those of high salt concentration, practically, do not shift toward the pumping well. For an ideal tracer, all isosalinity surfaces rise toward the pumping well. For brine, however, only iso-salinity surfaces of very low salinity upcone towards the pumping well. The decay process is lengthy; it takes a long time for the upconed saltwater to migrate back to the original horizontal transition zone prior to pumping. However, the wider transition zone caused by hydrodynamic dispersion can never return to the initial one. This indicates that once a pumping well is abandoned because of high salinity, it can be reused for groundwater utilization only after a long time.
\end{abstract}




\section{Introduction}

Brine or saltwater underlies the fresh water in many inland aquifers (e.g., Ma et al., 1997), with a transition zone separating them. The case of sea water intrusion in a coastal aquifer may serve as an example. Screened portions of pumping wells are often located within the fresh water zone that overlies the transition zone. We shall also use the term "interface zone" for this transition zone. During pumping, the transition zone upcones towards the screened portion of the well, and, the salinity of the pumped water increases. When the pumpage is constrained to be below some "critical pumping rate", the well may pump essentially fresh water (say, keeping salinity less than $2 \%$ of saltwater). If pumping rate is increased, the well will be salinized and the pumping will have to be shut-off. Following the shut-off, the upconed saline water mound decays, due mainly to gravity imbalance. The well may be restarted after the aquifer recovers from the influence of the previous pumping period. This pumping policy may increase the efficiency of fresh water pumping from the aquifer. This local upconing and decay processes can be superimposed on the regional seawater intrusion in a coastal aquifer (Bear et al., 2001).

Until recently, many sharp interface approximation models have been employed to investigate the problem of interface upconing, primarily in connection with sea water intrusion in coastal aquifers (Bear and Dagan, 1964; Bear, 1979; Dagan and Zeitoun, 1998). Up to a certain critical pumping rate, the sharp interface does not reach the well, and the latter continues to pump fresh water. At some critical pumping rate, the (assumed sharp) interface takes the shape of a very unstable cusp, and any increase in the pumping rate will immediately brings the interface, and with it seawater, into the pumping well. Under such conditions, the assumption of a sharp interface is no longer valid, and models based on it cannot be used. Analytical, experimental, and numerical methods have been employed to determine the position of the sharp interface and the critical pumping rate. A number of exact and approximate analytical solutions have been obtained for local upconing in the case of steady flow in homogeneous aquifers (Muskat, 1937; Strack, 1973; Bower et al., 1999). Dagan and Zeitoun (1998) developed an analytical solution for interface upconing in a stratified aquifer with random hydraulic conductivity. Hele-Shaw analogs (that model flow in a vertical plane) were employed to verify the approximate analytical solutions (Muskat, 1937; Bennett et al., 1968; Haubold, 1975). Numerical modeling has become a powerful tool for investigating upconing in field applications. Sahn (1973) developed a finite difference model for the simulation of the upconing problem. Pinder and Page (1976) obtained the local upconing under a pumping well by solving for the regional interface under an island. Reilly et al. (1987) applied a finite element model to estimate the maximum steady-state permissible discharge 
rate in an inland groundwater system. Kemblowski (1987) investigated the impact of the Duipuit-Forchheimer approximation on the simulation of upconing. He found that this approximation might produce large errors in estimating the upconing beneath a point drain, but produces good results for that below a river, which is wide relative to the thickness of aquifer. Sharp interface models neglect the effect of hydrodynamic dispersion on upconing, and they often overestimate the critical pumping rate.

The transition zone model for upconing takes into account hydrodynamic dispersion. Rubin and Pinder (1977) described upconing as a migration of a sharp interface perturbed by a small disturbance that results from dispersion. Wirojanagud and Charbeneau (1985) superposed the effect of hydrodynamic dispersion on a sharp interface solution. During the last two decades, a number of computer codes have been developed for simulating the coupled density-dependent flow and salt transport (Bear et al., 1999). For example, Diersch (1984) simulated a local upconing problem in cylindrical coordinate using the finite element code FEFLOW. Reilly and Goodman (1987) obtained solutions to a local upconing problem, using the SUTRA code. They compared their solutions with those obtained by Bernett et al. (1968), based on the sharp interface approximation, and found that the sharp interface model overestimates the critical pumping rate, although the 0.5 iso-concentration surface is close to the sharp interface configuration in the area away from the pumping well. Ma et al. (1997) applied the SWIFT-II code to an upconing problem of freshwater underlain by a layer of brine in an aquifer. Bear et al. (2001) used the FEAS-SWICA code to simulate seawater upconing to a pumping well in a regional coastal aquifer. Most of the previous research focused on the upconing problem for seawater intrusion with a small difference in density between pure saltwater and fresh water.

Different density differences may produce very different upconing phenomena. Oswald (1998) and Oswald et al. (2002) conducted a series of laboratory experiments on saltwater upconing using solutions with different salt concentrations in a three-dimensional cubical domain. The experimental results demonstrated that the saltwater upconing regime is very sensitive to the density of the salt solution. The same experimental results were also reproduced by Johannsen et al. (2002) using a numerical model.

The objective of this research is to explore the detailed mechanisms of flow and salt transport involved in the upconing problem and to investigate the effects on the upconing and decay processes of different density differences between the fresh and salt water and of the magnitude of transport parameters (dispersivity). We shall start in Sec. 2 by presenting the coupled density-dependent flow and salt transport model, based on the Eulerian-Lagrangian method, solved by the computer code FEAS-Brine. A brief description of the numerical 
schemes employed in FEAS-Brine is also presented in Sec. 3. The application to the transient upconing and decay processes beneath a pumping well in an axially symmetrical system is presented in Sec. 4.

\section{Mathematical Model}

The general mass balance equations for a aqueous liquid phase and for a dissolved solute component (salt) provide the governing equations for the strongly coupled densitydependent flow and salt transport (Bear and Verruijt, 1990; Bear and Bachmat, 1991). It is assumed that the generalized Darcy's law and a Fickian-type law are valid for the fluid's motion and for the dispersive flux, respectively. We do not include the cross-coupling terms in Darcy's law and dispersive flux for high concentration of brine, as suggested by Hassanizadeh and Leijnse (1988).

\subsection{Constitutive Equations}

A typical relationship between fluid density, $\rho$, pressure, $p$, and mass fraction of salt (mass of dissolved salt per unit mass of fluid), $\omega$, is:

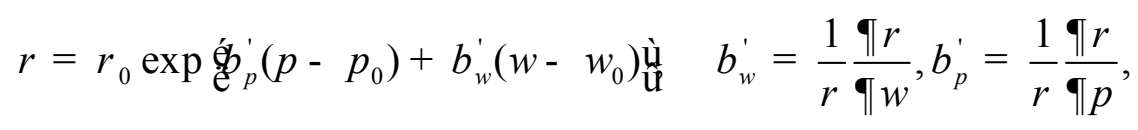

where $p=p_{0}$ and $w=w_{0}$ correspond to $r=r_{0}, \beta_{p}^{\prime}$ is the coefficient of fluid compressibility and $b_{w}^{\prime}$ is a density coefficient. The linearized approximation of (1) is

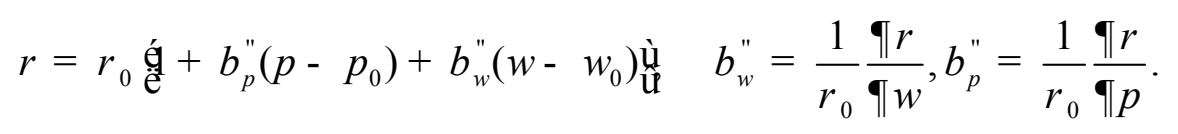

We shall assume that for the range of pressures considered here, $b_{w}^{\prime \prime}|\mathrm{D} w| ? b_{p}|\mathrm{D} p|$, so that we may employ the approximation:

$$
r=r_{0} \text { 宒 }+b_{w}^{\prime \prime}\left(w-w_{0}\right) \text { ù̀ }
$$

Freshwater $\left(r_{f w}\right)$ is often used for the reference fluid. We then have $w_{0}=w_{f w}$ and $r_{0}=r_{f w}$ and

$$
r=r_{f w} \stackrel{\mathrm{g}}{\mathrm{e}}+b_{w}^{\prime \prime}\left(w-w_{f w}\right) \text { प्̀े }
$$


Because in this work, we consider two fluid layers: a layer of low salt concentration (to which we refer as "freshwater") and a layer of high salt concentration (sea water or brine, to which we refer as "saltwater"), it is convenient to introduce a normalized mass fraction of salt in solution, $C\left(=\left(w-w_{f w}\right) /\left(w_{s w}-w_{f w}\right)\right)$, where $\omega_{f w}$ and $\omega_{s w}$ are the salt mass fractions of freshwater and of saltwater, respectively. We then have

$$
r=r_{f w}\left[1+b_{c} C\right] \quad b_{c}=b_{w}^{\prime \prime}\left(w_{s w}-w_{f w}\right),
$$

where $b_{c}$ is a density difference factor (DDF). Note that in what follows, we shall use the approximation expressed by (5), except in the definition of the specific storativity (see below), where we do take into account the effect of pressure on $r$. Also, because of the nonlinearity of the variable density problem, the salt content of the "freshwater" should be in the range, where its effect on the density is negligible. In this paper, we investigate the effect of the DDF on the upconing regime.

The fluid's dynamic viscosity, $\mu$, varies with the salt mass fraction, $w$, in the form:

$$
m=m_{f w} m_{r}=m_{f w}\left(1+1.85 w-4.1 w^{2}+44.50 w^{3}\right),
$$

which is based on experimental data from Weast (1989), with fitting by Lever and Jackson (1985) and Hassanizadeh and Leijnse (1988). $m_{f w}$ is the freshwater dynamic viscosity, and $m_{r}$ is the relative viscosity.

\subsection{Density-Dependent Flow Equation}

The general mass balance equation for the aqueous phase takes the form (Bear and Bachmat, 1991)

$$
\frac{\partial \phi \rho}{\partial t}=-\frac{\partial \rho q_{i}}{\partial x_{i}}+\left(\rho^{*} Q_{R}-\rho Q_{P}\right),
$$

with the injection and pumping through wells represented, symbolically, by

$$
\begin{aligned}
& \rho^{*} Q_{R}=\sum_{n} \rho_{n}^{*} Q_{R n}\left(\mathbf{x}_{n}, t\right) \delta\left(\mathbf{x}-\mathbf{x}_{n}\right), \\
& \rho Q_{P}=\sum_{m} \rho_{m} Q_{P m}\left(\mathbf{x}_{m}, t\right) \delta\left(\mathbf{x}-\mathbf{x}_{m}\right),
\end{aligned}
$$

where $x_{i}$ is the $i$ th component of the Cartesian coordinates, $\mathbf{x}, t$ is the time, $\phi$ is the porosity, $q_{i}$ is the $i$ th component of the specific discharge vector $\mathbf{q}, Q_{R n}$ and $\rho_{n}^{*}$ are the injection rate, and fluid's density of water injected through a well at point $\mathbf{x}_{n}$, respectively, $Q_{P m}$ and $\rho_{m}$ are 
the pumping rate and density of water pumped through a well at point $\mathbf{x}_{m}$. For the sake of simplicity, we shall, henceforth, refer to the aqueous liquid phase, as salt solution, or as "water".

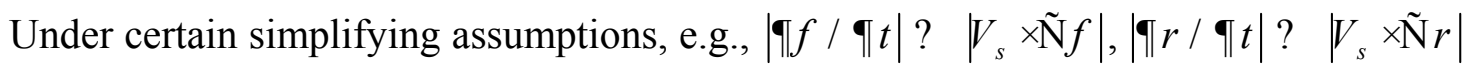
(Bear and Bachmat, 1991), the above mass balance equation for water can be rewritten as:

$$
f \frac{d r(p, w)}{d t}+r \frac{1}{1-f} \frac{\mathbb{\|} f}{\mathbb{\|} t}=-\frac{\mathbb{\|} r q_{r i}}{\mathbb{\|} x_{i}}+\left(r^{*} Q_{R}-r Q_{P}\right),
$$

in which $\mathbf{q}_{r}$ is the specific discharge relative to the solid, expressed by Darcy's law. We may rewrite this equation in the form:

$$
\rho S_{0 p} \frac{\partial p}{\partial t}+\rho \phi \beta_{c} \frac{\partial C}{\partial t}=-\frac{\partial \rho q_{r i}}{\partial x_{i}}+\left(\rho^{*} Q_{R}-\rho Q_{P}\right),
$$

where $S_{0 p}\left(=f b_{p}^{\prime}+a\right)$ is the specific storativity with respect to pressure changes, $\alpha(=-(1 /(1-\phi)) d(1-\phi) / d p)$ denotes the coefficient of solid matrix compressibility (with $\alpha=0$ for the incompressible material considered here), $b_{c}$ is the coefficient defined in (5).

The generalized Darcy's law for a variable density fluid flow takes the form:

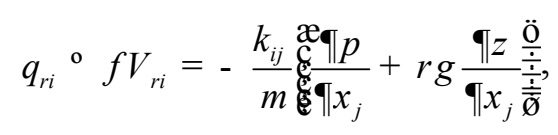

where $V_{r i}$ is the $i$ th component of the velocity vector (relative to the solid matrix), $k_{i j}$ is the permeability tensor, $\mu$ is the fluid's dynamic viscosity, which varies with mass fraction, $g$ is the gravitational acceleration, and $z$ is the vertical component of the Cartesian coordinate. After the introduction of a reference freshwater head, $h_{f}=h_{f}(x, y, z, t)$, as a primary variable:

$$
h_{f}=\frac{p}{\rho_{f w} g}+z
$$

Darcy's law can be rewritten as:

$$
q_{r i}=-\frac{K_{i j}^{f}}{\mu_{r}}\left(\frac{\partial h_{f}}{\partial x_{j}}+\beta_{c} C \frac{\partial z}{\partial x_{j}}\right)
$$


where $K_{i j}^{f}\left(=\rho_{f w} g k_{i j} / \mu_{f w}\right)$ is the reference hydraulic conductivity, related to the reference density $\rho_{f w}$ and to the reference dynamic viscosity $\mu_{f w}, \mu_{r}\left(=\mu / \mu_{f w}\right)$ is the relative dynamic viscosity.

By inserting Eqs. (11) and (12) into the mass balance equation, Eq. (9), we obtain the density-dependent flow equation:

$$
S_{0} \frac{\partial h_{f}}{\partial t}+\frac{\rho \phi \beta_{c}}{\rho_{f w}} \frac{\partial C}{\partial t}=\frac{\partial}{\partial x_{i}}\left(K_{i j}\left(\frac{\partial h_{f}}{\partial x_{j}}+\beta_{c} C \frac{\partial z}{\partial x_{j}}\right)\right)+\frac{\left(\rho^{*} Q_{R}-\rho Q_{P}\right)}{\rho_{f w}},
$$

where $K_{i j}\left(=\rho g k_{i j} / \mu\right)$ is the hydraulic conductivity, and $S_{0}\left(=\rho g\left(\phi \beta_{p}+\alpha\right)\right)$ is the specific storativity. Note that $K_{i j}$ and $S_{0}$ vary in both space and time, depending on the mass fraction of the salt solution.

\subsection{Salt Transport Equation}

The mass balance for the dissolved matter (salt) can be expressed, in terms of mass fraction of concentrated salt solution, assuming $w_{f w}=0$, in the form (Bear and Verruijt, 1990):

$$
\frac{\partial \phi \rho C}{\partial t}=-\frac{\partial}{\partial x_{i}}\left(\rho C q_{i}-\phi \rho D_{i j} \frac{\partial C}{\partial x_{j}}\right)+\left(\rho^{*} C^{*} Q_{R}-\rho C Q_{P}\right)
$$

with the injection and pumping through wells represented, symbolically, by

$$
\begin{aligned}
& \rho^{*} C^{*} Q_{R}=\sum_{n} \rho_{n}^{*} C_{n}^{*} Q_{R n}\left(\mathrm{x}_{n}, t\right) \delta\left(\mathrm{x}-\mathrm{x}_{n}\right), \\
& \rho C Q_{P}=\sum_{m} \rho_{m} C_{m} Q_{P m}\left(\mathrm{x}_{m}, t\right) \delta\left(\mathrm{x}-\mathrm{x}_{m}\right),
\end{aligned}
$$

where $C_{n}^{*}$ is the normalized mass fraction in the water injected through a well at point $\mathbf{x}_{n}, C_{m}$ is the normalized mass fraction in the water pumped through a well at point $\mathbf{x}_{m}$, and $D_{i j}$ is the coefficient of hydrodynamic dispersion.

Expanding the first-order derivatives in Eq. (14), and making use of the mass balance equation for water, Eq. (7), we obtain

$$
\phi \rho \frac{D C}{D t} \equiv \phi \rho\left(\frac{\partial C}{\partial t}+V_{i} \frac{\partial C}{\partial x_{i}}\right)=\frac{\partial}{\partial x_{i}}\left(\phi \rho D_{i j} \frac{\partial C}{\partial x_{j}}\right)+\rho^{*} Q_{R}\left(C^{*}-C\right) .
$$


where $D C / D t$ denotes the material derivative of $C$.

The density-dependent flow equation is coupled with the transport one by the two terms: $\frac{\partial}{\partial x_{i}}\left(K_{i j} \beta_{c} C \frac{\partial z}{\partial x_{j}}\right)$ (body-force term) and $\phi \beta_{c} \frac{\rho}{\rho_{f w}} \frac{\partial C}{\partial t}$, in addition to the coupling effect of the density variation on $K_{i j}$ and $S_{0}$. The transport equation is coupled with the flow equation by the velocity, which appears as part of the coefficient of hydrodynamic dispersion, and in the advective transport term. The two governing equations, (13) and (15), are, thus, nonlinear and coupled; they must be solved simultaneously.

The coefficient of hydrodynamic dispersion, $D_{i j}$, is the sum of the coefficient of mechanical dispersion and the coefficient of molecular diffusion in a porous medium, $D_{m}$. For an anisotropic medium (Bear, 1979; Bear and Verruijt, 1990):

$$
D_{i j}=a_{i j k m} \frac{V_{k} V_{m}}{V}+D_{m} \delta_{i j},
$$

where $a_{i j k m}$ is a typical component of the (fourth rank) dispersivity tensor, $V$ is the magnitude of the (average) velocity, and $\delta_{i j}$ denotes the Kronecker delta. In an isotropic porous medium with $x_{i}$ serving as principal directions, we have

$$
D_{i j}=a_{T} V \delta_{i j}+\left(a_{L}-a_{T}\right) \frac{V_{i} V_{j}}{V}+D_{m} \delta_{i j},
$$

where $a_{L}$ and $a_{T}$ are the longitudinal and transversal dispersivities, respectively.

\section{Numerical Model}

The mathematical flow model is solved by the Galerkin finite element method (FEM). Linear and bilinear basis functions are employed for a triangular mesh and for a quadrilateral mesh in two-dimensions, respectively. In three-dimensions, linear and bilinear basis functions are used for a tetrahedral and for a vertical triangular prism meshes. FEAS-Brine has been developed for the simulation of brine transport in three- and two-dimensional groundwater systems, by solving the coupled density-dependent flow and salt transport equations. This code is part of the code family, called FEAS, which has been verified in a number of benchmark cases (Zhou, 1999; Bensabat et al., 2000).

When solving the model, spurious velocity results from the inconsistency between the approximation of head gradient and fluid density (Voss and Souza, 1987; Herbert et al., 1988; 
Zhou et al., 2001). To avoid this spurious velocity in the vertical direction, in the FEM formulation of Darcy's law, the FEAS-Brine code uses an element-averaged fluid density for the body-force term. Thus, a linear or a multi-linear basis function is used for both the reference head and the mass fraction.

The advective-dispersive salt transport equation is solved in the Eulerian-Lagrangian framework. The Lagrangian mass fraction is derived by the adaptive pathline-based particle tracking algorithm presented by Bensabat et al. (2000). The Galerkin FEM is then used to solve the remaining dispersion equation. In the particle tracking algorithm, the tracking process is split along element boundaries on an inter-element basis. The sub-process within an element may be further refined along the particle's path by subdividing the travel time within this element into a number of travel time increments. Whether this in-element pathline-based refinement is needed or not depends on the complexity of the local velocity field. Tracking errors are controlled by practical criteria related to the rate of variation in the particle's velocity (in magnitude and direction). A bilinear spatial and temporal interpolation of particle velocity is used to avoid the error introduced by the stepwise temporal approximation used in most existing models. The efficiency of the particle tracking is improved by adapting the tracking time in each tracking step. This adaptation is based on two particle velocity indices and on their corresponding given criteria. The tracking process in regions where the velocity varies significantly is split into more tracking steps than in regions with smooth velocity variations. This adaptive particle-tracking algorithm performs very well in a complex flow field in a density-dependent flow and transport problem.

Thus, at each time step, there is a need to solve a set of non-linear equations. Time integration is performed using a first order approximation and an under-relaxation strategy, which tends to reduce the rate of convergence, but ensures a smoother, non-oscillating path to the solution. The linearization of the system is performed by using the Picard method. At each Picard iteration, the resulting set of linear equations is solved by applying a robust Incomplete Choleski Conjugate Gradient algorithm (Ajiz and Jennings, 1984, Axelson and Barker, 1984 and Barret et al., 1998). Time step size is determined in an adaptive way, depending on the convergence rate.

\section{Upconing and Decay Processes}

\subsection{Problem Statement}

We consider a problem of axially symmetrical upconing in a homogeneous, anisotropic confined aquifer, with unsteady pumpage from a partially penetrating well (see Figure 1). The 
$20 \mathrm{~m}$ long well screen is centered $10 \mathrm{~m}$ below the aquifer top. The well pumps water at a rate of $100 \mathrm{~m}^{3} / \mathrm{h}$. The well is shut-off when the normalized mass fraction of salt in the pumped water reaches the value of $2 \%$. Recharge of water occurs along the external boundary located at $x=2000 \mathrm{~m}$, where we assume that the effect of pumping is (practically) negligible. Along this vertical boundary, a hydrostatic pressure distribution is assumed to maintain, with the pressure (or the reference head) depending on the transient saline water density distribution. Initially, the aquifer contains freshwater in the upper portion $(120 \geq z \geq 22 \mathrm{~m})$ and saltwater (= seawater or brine) in the lower one $(z \leq 20 \mathrm{~m})$, with a $2 \mathrm{~m}$ thick transition zone. We have assumed that the thickness of the initial salt water portion of the aquifer $(20 \mathrm{~m})$ is such that (practically) it does not affect the resulting upconing. This problem is a modification of the upconing problem presented by Reilly and Goodman (1987) and Voss and Souza (1987). Unlike the specified $16 \mathrm{~m}$ thick initial transition zone and fixed saltwater mass fraction distribution on the outer radial vertical boundary in their model, we have modified these conditions to solution-dependent ones, keeping only the hydrostatic assumption. In addition, we allow for the influx of water and salt from the external vertical boundary, while their model allows influx also from the bottom boundary, by maintaining a constant pressure condition on it.

To complete the mathematical model, we need boundary and initial conditions, as well as model parameters. The top and bottom boundaries are impervious to both flow and transport, because the aquifer is confined. The axis at $x=0 \mathrm{~m}$ and $0 \leq z \leq 120 \mathrm{~m}$ is also a noflow boundary for both flow and transport. On the external vertical boundary, we assume that we are sufficiently far from the well so that the flow is always (essentially) horizontal, and, hence, the pressure (or reference head) distribution is always hydrostatic. This head distribution is updated with time based on the solution for the mass fraction distribution in each time step. For the salt transport, we assume that along this boundary, the iso-massfraction surfaces remain always horizontal (as they are initially). This means that for this vertical boundary, a zero mass fraction gradient is maintained normal to this boundary.

The aquifer medium and transport parameters are listed in Table 1. Three different groups of dispersivities are chosen to investigate the effect of hydrodynamic dispersion on the flow and transport regimes. Molecular diffusion is assumed negligible under the investigated field conditions, so that a narrow transition zone can be maintained in a region far away from the pumping well. The small dispersivities in Case B are chosen to show whether the FEASBrine code can produce a narrow transition zone with small or no numerical dispersion. The density difference factor in the basic case is $2.5 \%$, which is a common value for seawater. 
To investigate the effect of the density difference factor, $\beta_{c}$, on the upconing and decay processes, we also simulate the transient flow and transport under different $\beta_{c}$ values ranging from 0.0 (for ideal tracer), and 0.2 (for brine) with $\alpha_{L}=1.0 \mathrm{~m}$ and $\alpha_{T}=0.5 \mathrm{~m}$.

\subsection{Numerical Model}

Figure 2 shows a finite element mesh for the axially symmetrical problem. The mesh consists of $113 \times 60$ non-uniform rectangular finite elements and $114 \times 61$ nodes. The horizontal discretization increases from a spacing of $0.5 \mathrm{~m}$ close to the axis to a constant spacing of $25 \mathrm{~m}$ past a radius of $500 \mathrm{~m}$. Uniform discretization of $2 \mathrm{~m}$ is used for the vertical direction.

We simulate the coupled flow and salt transport problems for each of the combinations of transport parameter sets and the DDF. In each case, we simulate the transient upconing process until the well is shut off, when the normalized mass fraction of pumped water reaches $2 \%$, and the decay process following the well's shut-off. For each case, the total simulation time is 50 years (for brine case, the total simulation time is 80 years), and the time step size increases from the initial 0.01 day to 50 days. The time step size is also cut back to 0.01 day immediately following the well's shut-off to accurately simulate the decay process.

\subsection{Results and Discussion}

For the basic case with the dispersivities of $\alpha_{L}=1.0 \mathrm{~m}$ and $\alpha_{T}=0.5 \mathrm{~m}$ and $\beta_{c}=2.5 \%$, we obtain the entire transient process of reference head, velocity, and normalized mass fraction distributions in the 50 year period, which consists of the upconing period under pumping and decay period following the well's shut-off.

Initially, before pumping, we have a hydrostatic regime in the considered system. The reference head is determined by the boundary conditions on the external vertical boundary. The head at this boundary is selected as $10 \mathrm{~m}$ for the fresh water zone and $10.525 \mathrm{~m}$ $(=0.025 \times(0.5 \times 2 \mathrm{~m}+1.0 \times 20 \mathrm{~m}))$ at the bottom boundary caused by the density effects, noting that the normalized mass fraction distribution within the vertical element (transition zone) is linear because bilinear basis functions are used in the finite element model. Once we start pumping, a low reference head zone immediately occurs at the well screen caused by the pumping. At the same time, the head distribution beneath the pumping well also decreases, even in the transition zone and in the salt water zone (see Figure 3a). Thus, the pumping induces saltwater inflow at the external vertical boundary in both salt water zone and transition 
zone. The normalized mass fraction distribution at the initial time is uniform in the horizontal direction within the $2 \mathrm{~m}$ wide transition zone.

As the pumping proceeds, salt is transported towards the well by advection and dispersion in the shape of a saltwater cone or mound. The saltwater cone becomes higher in the vertical direction and wider in the horizontal direction as the upconing proceeds. The simultaneous change in the normalized mass fraction has an effect on the reference head distribution because the flow is density-dependent (or mass-fraction-dependent) and coupled with transport. The flow field undergoes a number of changes.

First, the reference head at a point within the saltwater cone increases because of the cumulative effect of the fluid's density above it. The head increase depends on the thickness of the transition zone above the point, and the normalized mass fraction distribution within the considered portion of the transition zone. Within the transition zone, the reference head is not distributed hydrostatically in the early time because there is a small vertical flow through this point. However, as the upconing proceeds with time, the thickness of transition zone beneath the pumping well increases, and the cumulative effect of the fluid's density at a point within the transition zone also increases (see Figure 4). In the horizontal direction, a smaller fraction of the total saltwater inflow from the external vertical boundary goes through the considered point, resulting in a smaller vertical flow at this point. To clearly demonstrate the transient processes of flow and transport in the vicinity of the well within the saltwater cone, we show in Figure 4 the simulated reference head and normalized mass fraction along two vertical lines ( $x=20,100 \mathrm{~m})$ and two simulation times ( $t=0.5,3$ years). At $t=0.5$ years, we can see the head difference between the two vertical lines, although the difference is small in the low portion of the transition zone. While such a difference decreases to essentially zero at $t=3$ years in the low transition zone, resulting in no or very small horizontal flow. The reference head in the upper portion of the two vertical lines decreases with time, because the pumping rate and the reference head within the fresh water zone at the external boundary remain unchanged. This indicates that less horizontal flow in the lower transition zone results in more horizontal flow to the pumping well occurring in the upper freshwater zone.

Within the transition zone, particularly in its low portion, the pressure distribution gradually approaches the hydrostatic one along the vertical, as shown in Figure 5. The reference head distribution (based on the assumed hydrostatic pressure distribution) at a point located within the low transition zone with $z$ coordinate along the vertical line of $x$ can be calculated by definition:

$$
h_{f}^{S}(x, z)=h_{f}^{*}(x, 0)-\int_{0}^{z} \beta_{c} C(x, \xi) d \xi
$$


where $h_{f}^{*}$ is the simulated reference head at the point on the bottom boundary, $h_{f}^{S}(x, z)$ is the hydrostatic reference head, and $C(x, \xi)$ is the simulated normalized mass fraction. In Figure 5, we can see that the simulated and the assumed hydrostatic reference head distributions are close to each other in the low portion of the transition zone, say, in the region of normalized mass fraction greater than 0.2 and less than 1.0. As the upconing proceeds, the hydrostatic zone expands in the vertical direction. For example, the hydrostatic zone is between $0 \mathrm{~m}$ and $30 \mathrm{~m}$ at 0.5 year, while it is between $0 \mathrm{~m}$ and $50 \mathrm{~m}$ at 3.0 years along the vertical line of $x=20 \mathrm{~m}$. Note that the saltwater zone under the saltwater cone quickly approach the hydrostatic (or quasi-hydrostatic) status (see Figures 4 and 5).

As upconing proceeds, the velocity in both vertical and horizontal direction decreases in the lower left corner region (defined as $[0,200] \times[0,22]$ and shown as Region A in Figure 1). After 1.2 years, a stagnant, or a small recirculating, flow cell gradually develops as a result of the increase in the reference head beneath the well. Figure 6 demonstrates the process of the development of the recirculating flow regime. A separation of flow occurs at $z=24 \mathrm{~m}$ along the inner radial boundary. The recirculating flow regime beneath the pumping well prevents mixed water at high mass fraction in the lower portion of transition zone from migrating upward to the well. The increase in salt mass in the upper portion of the transition zone, especially in the saltwater cone, has to be supplied from a region farther away from the central axis, widening the saltwater cone in the horizontal direction.

Coupled with the flow regime discussed above, the salt transport exhibits two different regimes in (a) the upconing region, say $0 \leq x \leq 800 \mathrm{~m}$, and (b) the far region away from the upconing region $(x>800)$ (see Figure 7$)$. In the far region, the transition zone widens with time in the vertical direction because of the transversal hydrodynamic dispersion, which depends on the essentially horizontal flow in this region. Along the $x$-direction, the transition zone becomes narrower because velocity decreases in the axially symmetrical system. On the external vertical boundary at $x=2000 \mathrm{~m}$, the transition zone also widens with time because the boundary conditions used for flow and transport allow for the updating of mass fraction and reference head.

In the upconing region, the salt transport depends on both advection and hydrodynamic dispersion, which is affected by both longitudinal and transversal dispersivity. The advective transport depends on the shape of the saltwater cone, which varies with time. As upconing proceeds, the saltwater cone becomes larger and the cone tends to be at hydrostatic regime. The saltwater cone at essentially hydrostatic regime behaves like a mound, while the advective salt transport behaves like the air current climbing to the peak of a mound. As the saltwater 
cone rises, the transition zone widens. The reference head and the mass fraction are consistent with each other because of the coupled flow and salt transport problem.

Figure 7 shows the normalized mass fraction distributions in the period of pumping and decay following the well's shut-off. As the pumping proceeds, the saltwater cone develops and becomes larger. The low iso-mass-fraction (iso-mf) surfaces rise locally towards the well. However, iso-mf surfaces of high mass fraction (e.g., the 0.8 iso-surface) do not move towards the well. The 0.5 iso-mf surface does not rise much, and then becomes stable after 3 years. Traditionally, this surface obtained by using the transition zone model is believed to represent the sharp interface obtained by a sharp interface model. If this is true, we can conclude that the sharp interface approximation overestimate the critical pumping rate or underestimate the critical rise for a given pumping rate. In the transition zone model, pumping fresh water can be maintained for a time without well salinization at a pumping rate lower than that predicted by a sharp interface model, mainly because of the involved hydrodynamic dispersion. Our findings are similar to those published by Reilly and Goodman (1987) and by Ma et al. (1997).

At $t=3.95$ years, the salinity in the pumped water reaches $2 \%$, and the well is shut off. The upconed interface, in the form of a local mound, undergoes decay and tends to decay back to the pre-pumping regime (see Figures 3 and 7). As decay proceeds, a large counterclockwise circulating flow caused by gravity imbalance is produced immediately after the well's shutoff (see Figure 8). This flow sweeps saltwater downward in the saltwater cone. It enables and facilitates the decay. On the other hand, the transition zone widens in the vertical direction out of the saltwater cone region. It takes very long time to completely return to the initial horizontal saltwater layer.

In terms of the width of the transition zone, the system will never reverse to the initial one. This long-time decay process and irreversible features are due to the absence of regional flow. Bear et al. (2001) found a reversible decay process following the well's shut-off in a coastal aquifer with regional freshwater flow to the sea.

\subsection{Effect of Dispersivity}

To demonstrate the effect of longitudinal and (mainly) transversal dispersivities on the coupled flow and salt transport, we simulate the entire transient processes of upconing and decay within 50 years for Cases $\mathrm{B}$ and $\mathrm{C}$ for the density difference factor of $2.5 \%$. The very small transversal dispersivity in Case B is used to demonstrate whether the FEAS-Brine code can produce a narrow transition zone with small or no numerical dispersion. Because different flow and transport regimes are obtained in the same simulation time for different values of dispersivity (Cases A, B and C), we compare the transport regimes among the three cases at 
the simulation time just before well's shut-off (when the pumped water contains $2 \%$ salt water) and at 50 years.

Figure 9 presents the normalized mass fraction distributions in Cases $\mathrm{B}$ and $\mathrm{C}$ when the well shuts off, and at 50 years. In Cases A, B, and C, the well is shut off at 3.95, 11.35, and 2.55 years, respectively. This indicates that the transversal (and longitudinal) dispersivity has a significant effect on the duration of well pumping for freshwater utilization. The smaller the dispersivities, the longer the well pumps fresh water. For example, the pumping duration in Case B is about four times that of Case A, while the transversal dispersivity in Case B is 25 times smaller than that in Case A. Figure 10a shows the breakthrough curves of normalized mass fraction in the pumped water for the three cases. We can see that in Case B, the breakthrough curve exhibits the slowing-down of the rate of increase (with respect to time) in the mass fraction of the pumped water. The conclusion on the pumping duration implies that the well can pump fresh water longer in a rather homogeneous aquifer than in a highly heterogeneous aquifer, because of the macrodispersivity (Dagan, 1989; Gelhar, 1993), when both aquifers have the same flow and transport properties (e.g., $\alpha_{L}$ and $\alpha_{T}$ ).

The transition zone is different in different cases in terms of (a) its width in the far region, and (b) the saltwater cone shape in the upconing region. The transition zone is very narrow in Case B because of the small transversal dispersivity used. In the far region, the flow is essentially horizontal, and the width of the transition zone is dominated by the transversal dispersivity. In Case $\mathrm{C}$, the largest transversal dispersivity produces the widest transition zone. The wide transition zone can shorten the pumping duration because of the shorter distance for salt particles to arrive at the well. Regarding the shape of the saltwater cone, we can see (from the shape of 0.02 iso-surface) that the entire cone in the horizontal cross-section is narrower in Case B than In Case C. The smaller radius of the saltwater cone in the cross section produces higher mass fraction in the center of the cone. For example, the 0.2 iso-mf surface reaches the well in Case B, while it reaches at $\mathrm{z}=72 \mathrm{~m}$ in Case $\mathrm{C}$. This is understandable in that the total salt flux discharged by the well should be identical at the well's shut-off for different cases (say, 2\% of normalized mass fraction in pumped water). A narrow cross-section of mixed water will require a higher mass fraction along the cross section. At 50 years, after a long time decay process, different transition zones are obtained for different cases (see Figures 7 and 9). The decay, or recovery, process takes a shorter time in Case B than in Case C. The narrow transition zone remains unchanged in Case $\mathrm{B}$, while the transition zone at 50 years becomes wider in both Cases $\mathrm{A}$ and $\mathrm{C}$ than that at the well's shut off time. For all the three cases, the decay process is slow and requires a very long time to completely recover. In summary, an increase in dispersivity, especially the transversal one, leads to a wider transition zone, a flatter 
salinity cone, an earlier well shut-off, and a longer period of decay of the upconed saltwater cone.

The obtained narrow transition zone in Case B demonstrates that the FEAS-Brine code can be used to simulate transport with small or no numerical dispersion. The reason for this advantage of the code is that the advective-dispersive transport equation is solved in the Eulerian-Lagrangian framework in two steps: (1) the Lagrangian mass fraction is derived by the adaptive pathline-based particle tracking algorithm (Bensabat et al., 2000), and (2) the remaining dispersion equation is then solved by the Galerkin FEM. The particle tracking algorithm used can accurately track particles within a complicated flow field by adaptively cutting the simulation time step size into a number of tracking time steps. This code does not suffer from the instability constraint of the Peclet number in the advection-dominated transport in the vicinity of the well.

\subsection{Effect of Density Difference Factor, $\beta_{c}$}

To investigate the effect of a density difference between the fresh water and the salt water (seawater or brine) on the upconing and decay process, we study the effect of the density difference factor, $\beta_{c}$. We conduct a sensitivity analysis by running three cases with different $\beta_{c}$ values $\left(\beta_{c}=0.0,0.025,0.2\right)$. The dispersivities used are $\alpha_{L}=1.0 \mathrm{~m}$ and $\alpha_{T}=0.5 \mathrm{~m}$. We compare the normalized mass fraction distributions in the three cases at the simulation time of well's shut-off (see Figures 11 and 7c). In the case of $\beta_{c}=0.0$ (representing an ideal tracer), the transient solute mass fraction distributions do not affect the fluid's flow, and the flow and transport are uncoupled. The pumping duration is 452 days, much less than 3.95 years in the case of $\beta_{c}=0.025$. This indicates that the density effect can help retard the solute transport toward the well. Unlike in the case of $\beta_{c}=0.025$, the iso-mf surfaces of both low and high mass fractions upcone towards the pumping well, resulting in the earlier shut-off of the well. Although the dispersivities used in both cases are the same, the transition zone at the time of well's shut-off behaves differently: the transition zone is narrower in the $\beta_{c}=0.0$ case than the $\beta_{c}=0.025$ case. However, the transition zone in Figure 11a ( $t=452$ days) is close to that in Figure $7 \mathrm{a}$ ( $t=1$ year), indicating that the transition zone away from the solute cone depends primarily on the dispersivities because the flow is essentially horizontal flow.

The most interesting comparison of the normalized mass fraction distributions is between the cases of $\beta_{c}=0.025$ (seawater) and $\beta_{c}=0.20$ (brine) (see Figures $7 \mathrm{c}$ and $11 \mathrm{~b}$ ). In the latter case, the well can pumps fresh water (in terms of $2 \%$ mass fraction of brine, rather 
than absolute salt mass fraction) for 80 years until the normalized mass fraction of the pumped water increases up to $2 \%$. The saltwater mound is composed of only low mass fraction fluid $(C<0.2)$, while the iso-mf surfaces of high mass fraction $(C>0.2)$ are almost horizontal straight lines (= horizontal surfaces), indicating that the salt mass migrating toward the well comes from the upper portion (with smaller mass fraction) of the transition zone. This indicates that the heavier brine in the lower portion tends to stay there (practically) without responding to the pumping. The transition zone is much wider and the saltwater mound is also much wider in terms of its radius. Therefore, the density difference factor plays a key role in the coupled density-dependent flow and transport and the difference between the weakly (seawater) and strongly coupled (brine) flow and transport is significant.

These findings of the effects of density difference factor are similar to those obtained in a series of laboratory experiments conducted in a three-dimensional cubical domain, referred to as "saltpool", by Oswald (1998), Oswald, et al. (2002), and Johannsen et al. (2002). In their phase 3 experiments (see Johannsen et al., 2002, Fig. 1), the saltwater initially takes the form of a horizontal layer below the freshwater in the cube. Freshwater was then injected into the system through an upper inflow hole, and mixed water was displaced out of this system through an upper discharge hole located at the diagonal line with the inflow hole. The entire transient saltwater distribution was recorded as a benchmark problem for densitydependent flow and transport. Two saltwater solutions with different salt-mass fractions ( $\omega=0.01,0.10)$ were used. Johannsen et al. (2002) numerically reproduced the saltwater upconing problem conducted in the laboratory experiments for the two selected salt solutions. In the case of $\omega=0.10$, the iso-mf surfaces of high normalized mass fractions (e.g., 0.5 and $0.9)$ lie horizontally at the transition zone, while only the mixed water with lower mass fractions was discharged out of the system (see Oswald, et al., 2002; Johannsen et al., 2002, Figure 6).

For water supply, the water quality in the pumped water may be based on the absolute mass fraction of salt, which is independent of salt mass fraction of the saltwater. For this purpose, we can also compare the simulation results for the seawater $\left(\beta_{c}=0.025\right)$ and brine $\left(\beta_{c}=0.20\right)$. From the breakthrough curve of normalized mass fraction in the pumped water shown in Figure 10b, we can see the shut-off time is at about 3.90 years, at which the pumped water contains $0.0025 \%$ mass fraction of brine, having the same absolute salt mass fraction as $2 \%$ mass fraction of seawater. The pumping duration in both cases is similar. However, the mass fraction contours at the well's shut-off time in both cases are different. In the brine case, the saltwater cone has a larger radius for the same absolute salt mass fraction as in the seawater case. The brine zone of high normalized mass fraction is always at hydrostatic status 
because of higher density effect. The salt transport happens in the upper thin layer of very low normalized mass fraction in the transition zone due to hydrodynamic dispersion at the early time. Unlike the upconing beneath the pumping well in the seawater case, small advective transport of salt occurs beneath the well in the brine cases. More salt has to be transported from far region away from the well vicinity.

\section{Conclusions}

Upconing of saltwater underlying the fresh water zone in many aquifers can cause the contamination/salinization of fresh water sources. The salinity of the pumped water depends on the well's settings (pumping rate and the well's screen), on the fresh water flow regime, on the density difference factor, and on the dispersivities. The code FEAS-Brine, developed for simulating the strongly coupled density-dependent flow and salt transport, was used to simulate the transient processes of the upconing and mound decay following the well's shutoff in an axially symmetrical system. Sensitivity analysis was conducted with respect to the longitudinal and transversal dispersivities, and the density difference factor. The simulation results for seawater of moderate value of density difference factor show that local upconing happens only for iso-mass-fraction surfaces of low normalized mass fractions, while those of high mass fractions practically do not shift toward the pumping well. This is particularly true for brine with a high density difference factor of 0.2 , showing that the saltwater mound contains mixed water of very small normalized mass fraction, while all other mixed water lie horizontally in the lower portion of the transition zone. It was found that the density effect retards the solute transport toward the pumping well and prolongs the pumping duration prior to salinization. The findings on local upconing of brine are consistent with those in laboratory experiments conducted in a three-dimensional cubical domain (Oswald, et al., 2002; Johannsen et al., 2002). The findings for brine imply that the traditional sharp interface model cannot produce a reasonable approximation for brine transport, because the 0.5 iso-massfraction surface of brine remains (practically) horizontal, rather than upconing toward the well. This is contrary to the assumption underlying many investigations that the 0.5 isoconcentration surface is close to the sharp interface solution.

It was found that the upconing process is sensitive to both transversal dispersivity and longitudinal dispersivity. A small value of dispersivity produces a very narrow transition zone and longer pumping duration. The obtained narrow transition zone demonstrates that the FEAS-Brine code can be used for transport simulation with small or no numerical dispersion. This may be attributed to the Eulerian-Lagrangian framework used for solving the advectivedispersive salt transport and adaptive pathline-based particle tracking algorithm used in this 
code. This code does not suffer from the instability constraint of Peclet number in an advection-dominated problem.

\section{Reference}

Axelsson, O. and Barker, V. A.: 1984, Finite element solution of boundary value problem, theory and computation, Academic Press, Orlando.

Ajiz, M. A. and Jennings, A.: 1984, A robust incomplete Choleski conjugate gradient algorithm, Int. J. Numer. Methods Eng., 20, 949-966.

Barrett, R., Berry, M., Chan, T. F., Demmel, J., Donato, J. M., Dongarra, J., Eijkhout, V., Pozo, R., Romine, C., and Van der Vorst, H.: 1994, Templates for the solution of linear systems: building blocks for iterative methods, SIAM, Philadelphia.

Bear, J. and Dagan, G.: 1964, Moving interface in coastal aquifers, Proc. A.S.C.E. 99(HY4), 193-215.

Bear, J.: 1979, Hydraulics of groundwater, McGraw-Hill, New York.

Bear, J. and Verruijt, A.: 1990, Modeling groundwater flow and pollution, Kluwer Academic Publishers, Dordrecht.

Bear, J., and Bachmat, Y: 1991, Introduction to modeling of transport phenomena in porous media, Kluwer academic publishers, Dordrecht.

Bear, J., Cheng, A. H-D., Sorek, S., Ouazar, D., and Herrera I. (Eds.):1999, Seawater intrusion in coastal aquifers - concepts, methods and practices, Kluwer Academic Publishers, Dordrecht.

Bear, J., Zhou, Q-L., and Bensabat, J.: 2001, Three dimensional simulation of seawater intrusion in heterogeneous aquifers: application to the coastal aquifer of Israel, in the proceedings of the First International Conference on Saltwater Intrusion and Coastal Aquifers-Monitoring, Modeling, and Management, Essaouira, Morocco, April 23-25.

Bennett, G.D., Mundorff, M. J., and Hussain, S. A.: 1968, Electric-analog studies of brine coning beneath freshwater wells in the Punjab Region, West Pakistan, U.S. Geol. Surv. Water-Supply Paper, 1608-J.

Bensabat, J., Zhou, Q-L., and Bear, J.: 2000, An adaptive pathline-based particle tracking in the Eulerian-Lagrangien finite element method, Adv. Water Resour., 23(4), 383-387. 
Bower, J.W., Motz, L.H., and Durden, D.W.: 1999, Analytical solution for determining the critical condition of saltwater upconing in a leaking artesian aquifer, J. Hydrol., 221, 4354.

Dagan, G., 1989: Flow and transport in porous formations, Springer-Verlag, Berlin.

Dagan, G. and Zeitoun, D.G.: 1998: Free-surface flow toward a well and interface upconing in stratified aquifers of random conductivity, Water Resour. Res., 34(11), 3191-3196.

Diersch, H. J., Prochnow, D., and Thiele, M.: 1984, Finite element analysis of dispersionaffected saltwater upconing below a pumping well, Applied Mathematical Modeling, 8(5), 305-312.

Gelhar, L.W., 1993: Stochastic subsurface hydrology, Prentice Hall, Englewood Cliffs.

Hassanizadeh, S. M. and Leijnse, T.: 1988, On the modeling of brine transport in porous media, Water Resour. Res., 24, 321-330.

Haubold, R. G.: 1975, Approximation for steady interface beneath a well pumping freshwater overlying saltwater, Ground Water, 13(3), 254-259.

Herbert, A. W., Jackson, P., and Lever, D. A.: 1988, Coupled groundwater flow and solute transport with fluid density strongly dependent upon concentration, Water Resour. Res., 24, 1781-1795.

Kemblowski, M.: 1987, The impact of the Dupuit-Forchheimer approximation on saltwater intrusion simulation, Ground Water, 25(3), 333-336.

Johannsen K., Kinzelbach, W., Oswald, S., and Wittum G.: 2002, The saltpool benchmark problem - numerical simulation of saltwater upconing in a porous medium, Adv. Water Resour., 25, 335-348.

Lever, D. A. and Jackson, C. P.: 1985, On the equations for the flow of a concentrated salt solution through a porous medium, Harwell Rep. AERE-R. 11765, Her Majesty's Stationary Off., London.

Ma, T-S., Sophocleous, M., Yu, Y-S., and Buddemeier, R.W.: 1997, Modeling saltwater upconing in a freshwater aquifer in south-central Kansas, J. Hydrol.,201, 120-137.

Muskat, M.: 1937, The flow of homogeneous fluids through porous media, McGraw-Hill, New York.

Oswald, S.:1998, Density-driven flow in porous media: three-dimensional experiments and modeling, Ph.D. Thesis, Institute of Hydromechanics and Water Resources Management, ETH Zurich, Switzerland. 
Oswald, S. E., Scheidegger, M. B., and Kinzebach, W., :2002, Time-dependent measurement of strongly density-dependent flow in a porous medium via nuclear magnetic resonance imaging, Transport in Porous Media, 47(2), 169-193.

Pinder, G. F., and Page, R. H.: 1977, Finite element simulation of salt water intrusion on the South Fork of Long Island, In: Finite Elements in Water Resources, Proc. $1^{\text {st }}$ int. Conf. Finite Elements in Water Resources, Pentech, London, 2.51-2.69.

Reilly, T. E. and Goodman, A. S.: 1987, Analysis of saltwater upconing beneath a pumping well, J. Hydrol., 89, 169-204.

Reilly, T. E., Frimpter, M. H., LeBlanc, D. R., and Goodman, A. S.: 1987, Analysis of steadystate salt-water upconing with application at Truro well field, Cape Code, Massachusatts, Ground Water, 25(2), 194-206.

Rubin, H., and Pinder, G. F.: 1977, Approximate analysis of upconing, Adv. Water Resour., 1(2), 97-101.

Sahni, B. M.: 1973, Physics of brine upconing beneath skimming wells, Ground Water, 11(1), 19-24.

Strack, O. D. L.: 1972, Some cases of interface flow towards drains, J. Eng. Math., 6, 175191.

Voss, G. I., and Souza, W. R.: 1987, Variable density flow and solute transport simulation of regional aquifers containing a narrow freshwater-saltwater transport zone, Water Resour. Res., 10, 1851-1866.

Weast R. C. (Ed.): 1989, Handbook of chemistry and physics, 70th edition, Chemical Rubber Publishing Company (CRC), Boca Raton, Florida.

Wirojanagud, P., and Charbeneau, R. J.: 1985, Saltwater upconing in unconfined aquifers, $J$. Hydraul. Eng., A.S.C.E., 111(3), 417-434.

Zhou, Q.-L.: 1999, Modeling seawater intrusion in coastal aquifers, Ph.D. thesis, TechnionIsrael Institute of Technology.

Zhou, Q-L., Bensabat, J., and Bear, J.: 2001, Accurate calculation of specific discharge in heterogeneous porous media, Water Resour. Res., 37(12), 3057-3069. 
Figure 1. Initial and boundary conditions for the upconing problem in an axially symmetrical system.

Figure 2. Finite element mesh for the upconing problem $(113 \times 60$ rectangular elements $)$.

Figure 3. Reference head distributions at (a) initial time with pumping, and (b) at 3.95 years before well's shut-off, (c) 5 years and (d) 20 years in the decay period in the basic case with $\alpha_{L}=1.0 \mathrm{~m}$ and $\alpha_{T}=0.5 \mathrm{~m}$ and $\beta_{c}=2.5 \%$.

Figure 4. Vertical distributions of simulated reference head and normalized mass fraction at $x=20 \mathrm{~m}$ and $100 \mathrm{~m}$ at 0.5 years and 3 years.

Figure 5. Vertical distribution of the simulated reference head (by solid lines) and the calculated head ( by symbols) using hydrostatic status assumption at two different times at $\mathrm{x}=$ $20 \mathrm{~m}$.

Figure 6. Development of a recirculating flow cell in Case $A$ in the region of $[0,200 m] \times[0,22 m]$.

Figure 7. Transient distributions of normalized mass fraction in Case $\mathrm{A}$ ( $\alpha_{L}=1.0 \mathrm{~m}$ and $\alpha_{T}=0.5 \mathrm{~m}$ ). The left-side panel indicates results under well pumping, while the right-side one indicates results during the period of decay following the well's shutoff.

Figure 8. Velocity field at $t=5$ years during the period of decay following the well's shutoff in Case A.

Figure 9. The normalized mass fraction distributions at (a) 11.35 years and (b) 50 years in Case B $\left(\alpha_{L}=0.2 \mathrm{~m}\right.$ and $\left.\alpha_{T}=0.02 \mathrm{~m}\right)$, and (c) 2.55 years and (d) 50 years in Case C $\left(\alpha_{L}=10 \mathrm{~m}\right.$ and $\left.\alpha_{T}=1.0 \mathrm{~m}\right)$.

Figure 10. The breakthrough curves of the normalized mass fraction in the pumped water in the case of (a) seawater ( $\left.\beta_{c}=0.025\right)$ and (b) brine $\left(\beta_{c}=0.20\right)$. 
Figure 11. The normalized mass fraction distributions of (a) a tracer $\left(\beta_{c}=0.0\right)$ at 452 days of pumping, and (b) brine ( $\left.\beta_{c}=0.20\right)$ at 80 years of pumping in the case of $\alpha_{L}=1.0 \mathrm{~m}$ and $\alpha_{T}=0.5 m$. 
Table 1. Model parameters for the upconing problem.

\begin{tabular}{|c|c|c|c|}
\hline \multicolumn{2}{|c|}{ Parameter } & \multicolumn{2}{|c|}{ Value } \\
\hline \multicolumn{2}{|c|}{ Porosity } & \multicolumn{2}{|l|}{$\phi=0.2$} \\
\hline \multicolumn{2}{|c|}{ Horizontal permeability } & \multicolumn{2}{|c|}{$k_{x}=2.56 \times 10^{-11} \mathrm{~m}^{2}$} \\
\hline \multicolumn{2}{|c|}{ Vertical permeability } & \multicolumn{2}{|c|}{$k_{z}=1.0 \times 10^{-11} \mathrm{~m}^{2}$} \\
\hline \multicolumn{2}{|c|}{ Dynamic viscosity } & \multicolumn{2}{|c|}{$\mu=1.0 \times 10^{-3} \mathrm{~kg} / \mathrm{ms}$} \\
\hline \multicolumn{2}{|c|}{ Gravity acceleration } & \multicolumn{2}{|c|}{$g=9.81 \mathrm{~m} / \mathrm{s}^{2}$} \\
\hline \multicolumn{2}{|c|}{ Pumping rate } & \multicolumn{2}{|c|}{$Q_{w}=2400 m^{3} / d$} \\
\hline \multicolumn{2}{|c|}{ Density of pure freshwater } & \multicolumn{2}{|c|}{$\rho_{f w}=1000 \mathrm{~kg} / \mathrm{m}^{3}$} \\
\hline \multirow[t]{3}{*}{ Case A } & Longitudinal dispersivity & \multicolumn{2}{|l|}{$\alpha_{L}=1 m$} \\
\hline & Transversal dispersivity & \multicolumn{2}{|l|}{$\alpha_{T}=0.5 m$} \\
\hline & Molecular diffusion coefficient & \multicolumn{2}{|l|}{$D_{m}=0 m^{2} / s$} \\
\hline Case B & $\alpha_{L}=0.2 m$ & $\alpha_{T}=0.02 m$ & $D_{m}=0 m^{2} / s$ \\
\hline Case $\mathrm{C}$ & $\alpha_{L}=10 m$ & $\alpha_{T}=1 m$ & $D_{m}=0 m^{2} / s$ \\
\hline
\end{tabular}




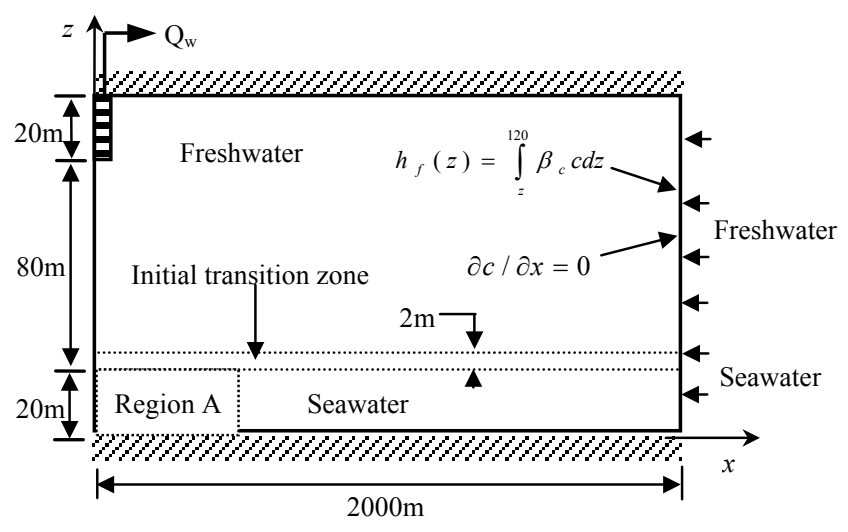

Figure 1. Initial and boundary conditions for the upconing problem in an axially symmetrical system.

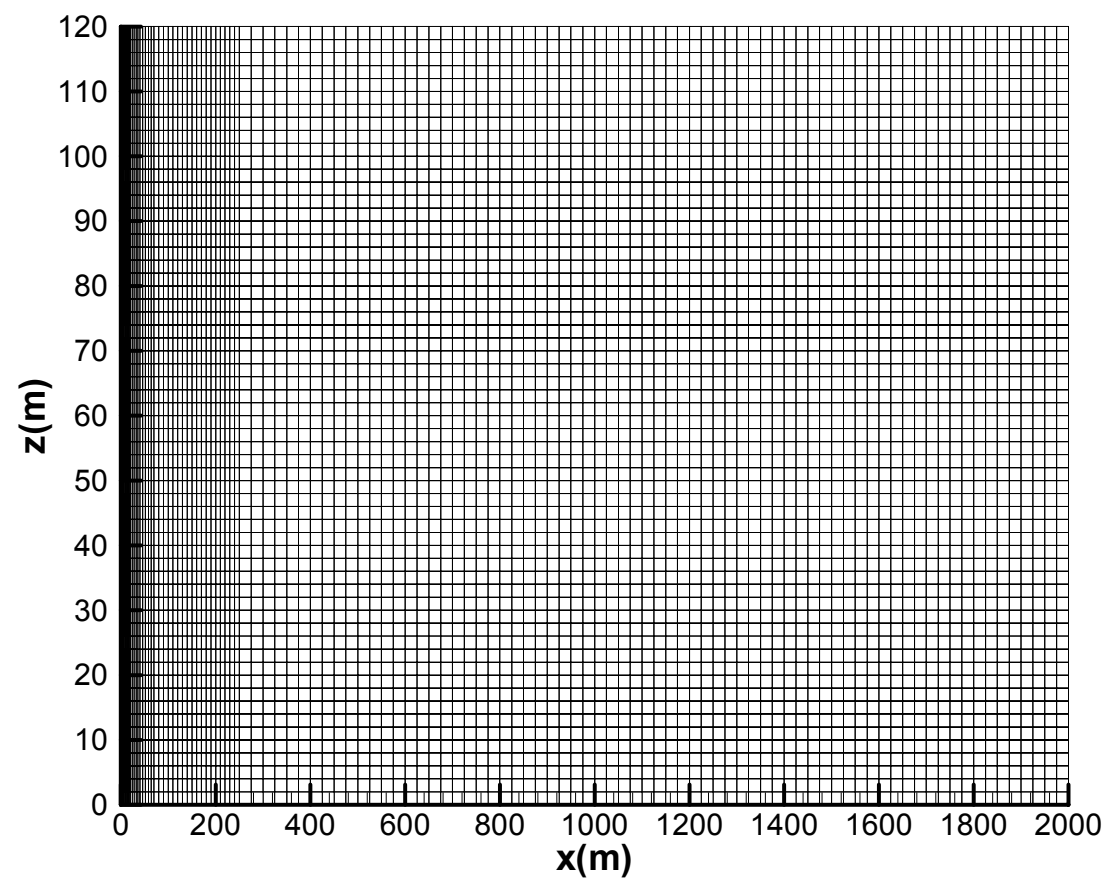

Figure 2. Finite element mesh for the upconing problem $(113 \times 60$ rectangular elements $)$. 

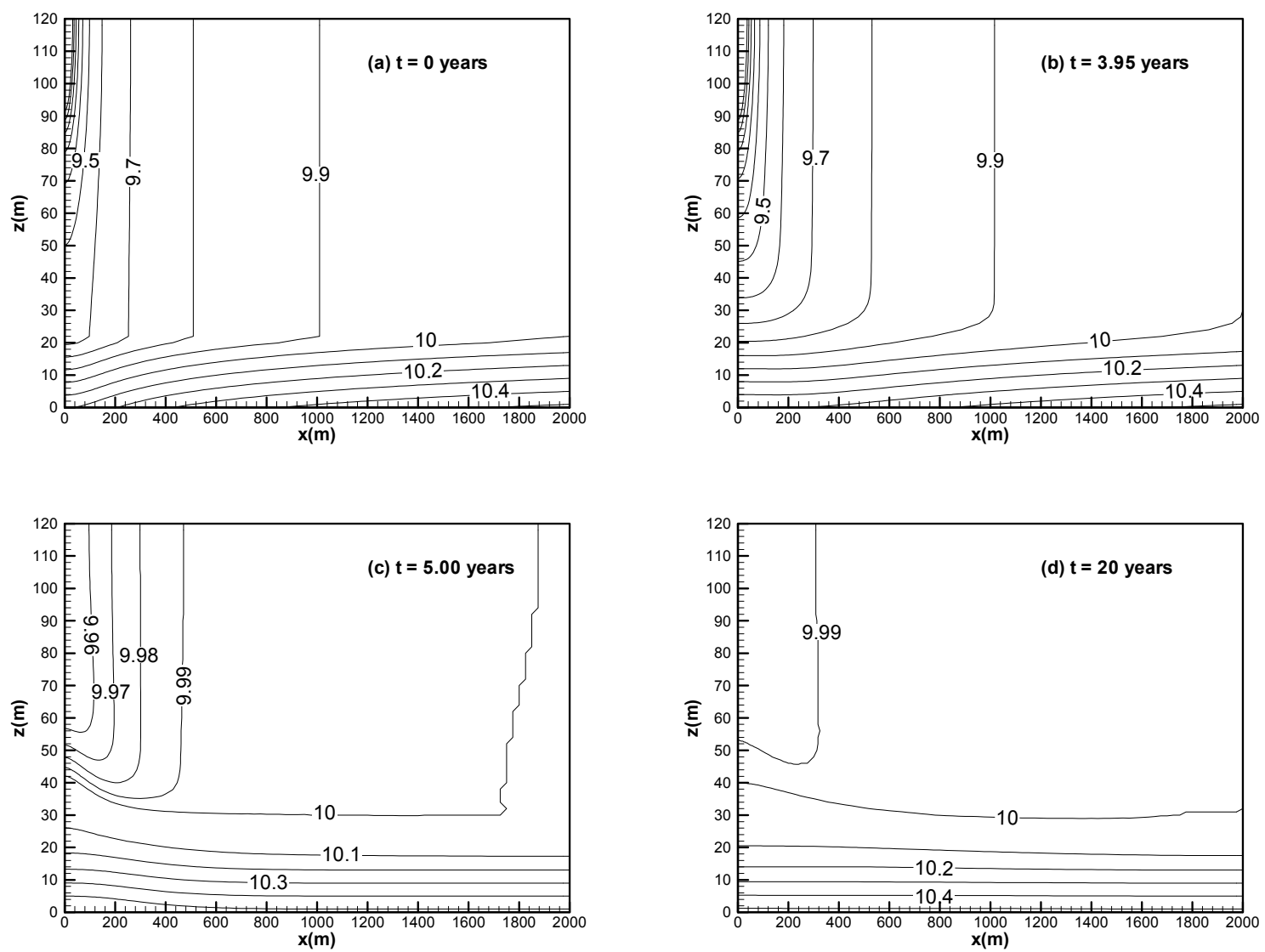

Figure 3. Reference head distributions at (a) initial time with pumping, and (b) at 3.95 years before well's shut-off, (c) 5 years and (d) 20 years in the decay period in the basic case with $\alpha_{L}=1.0 \mathrm{~m}$ and $\alpha_{T}=0.5 \mathrm{~m}$ and $\beta_{c}=2.5 \%$. 

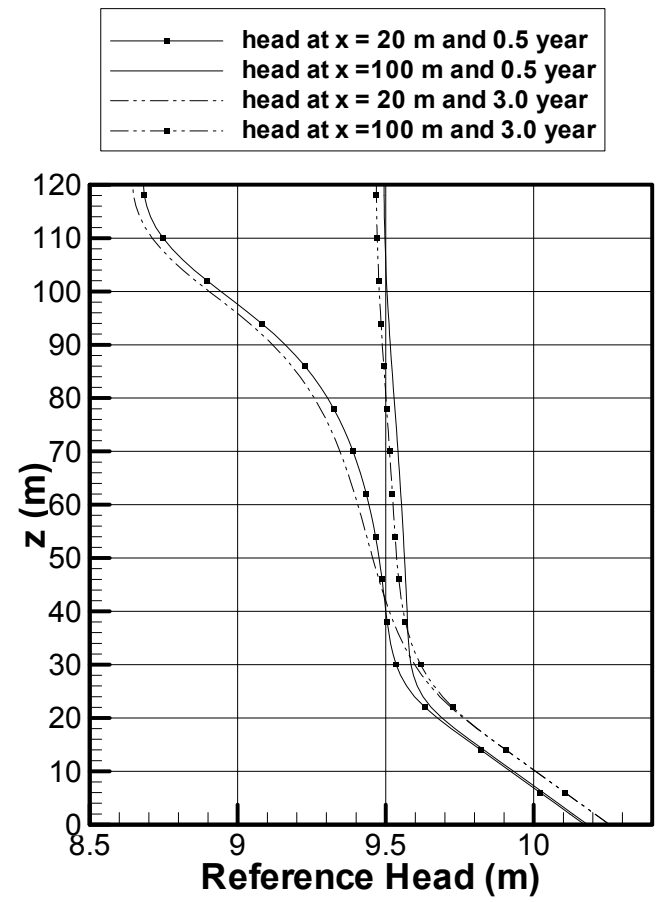
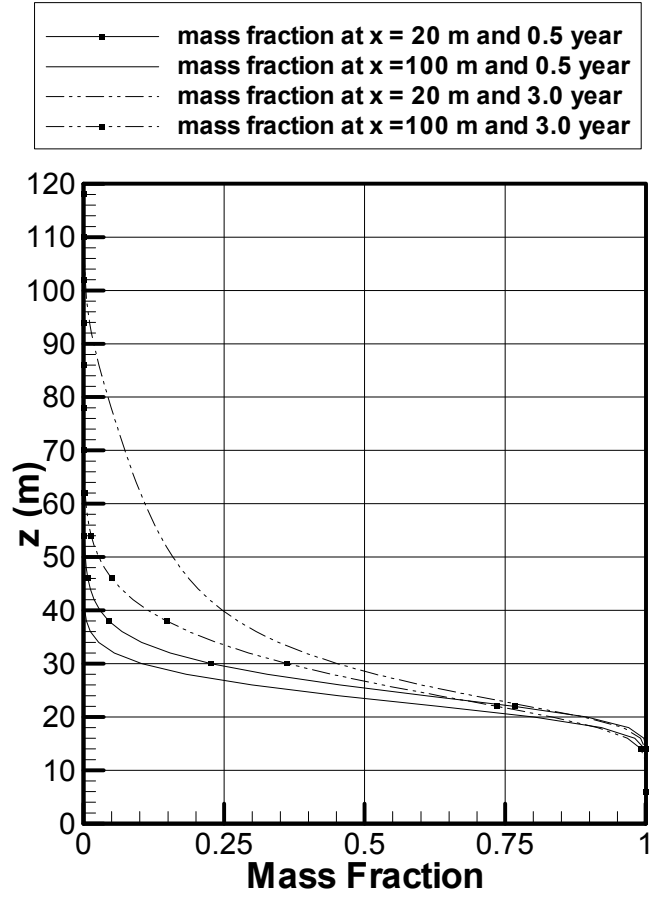

Figure 4. Vertical distributions of the simulated reference head and normalized mass fraction at $x=20 \mathrm{~m}$ and $100 \mathrm{~m}$ at 0.5 years and 3 years. 

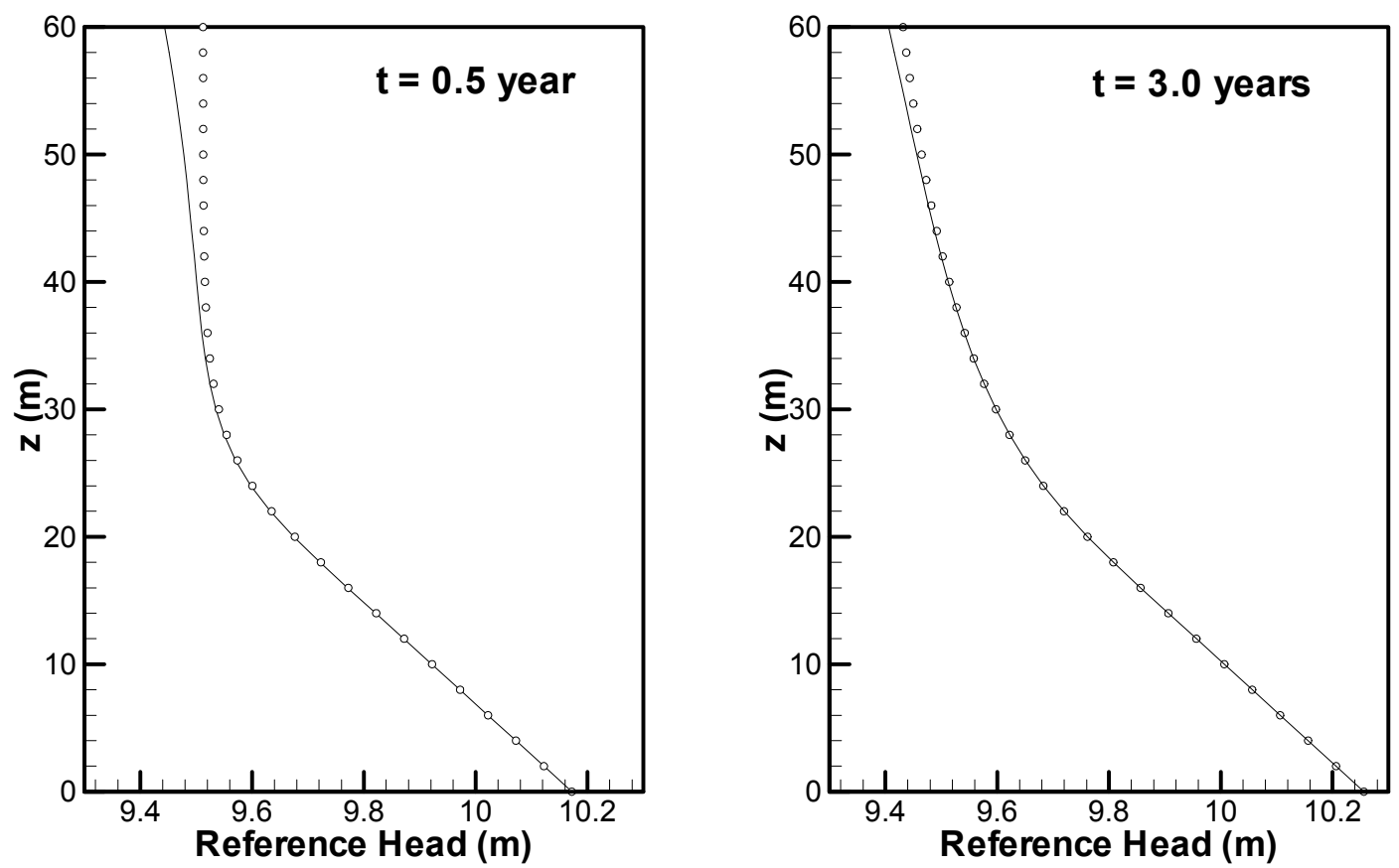

Figure 5. Vertical distribution of the simulated reference head (by solid lines) and the calculated head (by symbols) using hydrostatic status assumption at two different times at $\mathrm{x}=20 \mathrm{~m}$. 


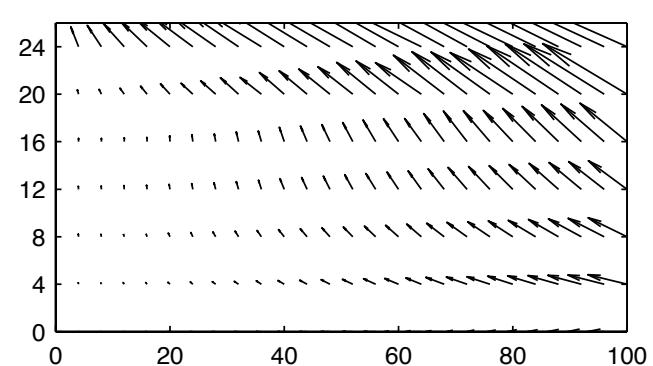

(a) at 1 year

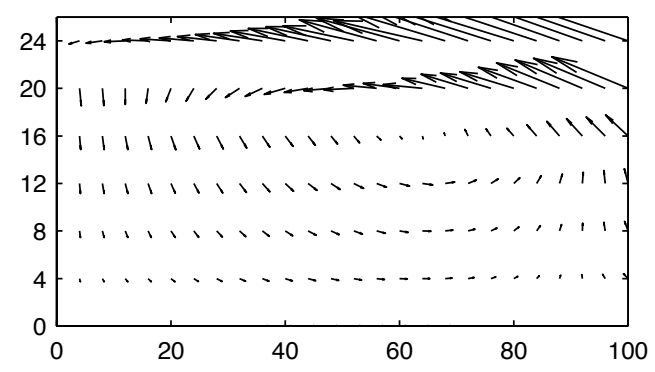

(b) at 2 years

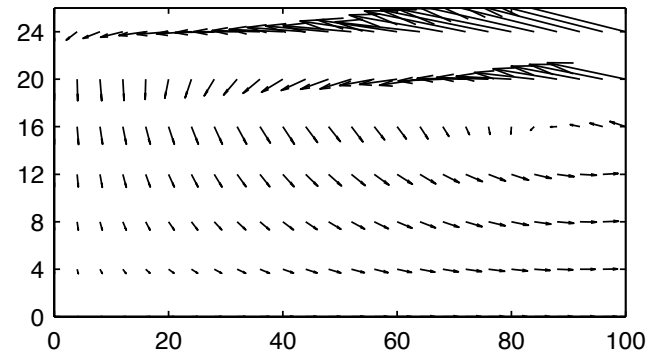

(c) at 3 years

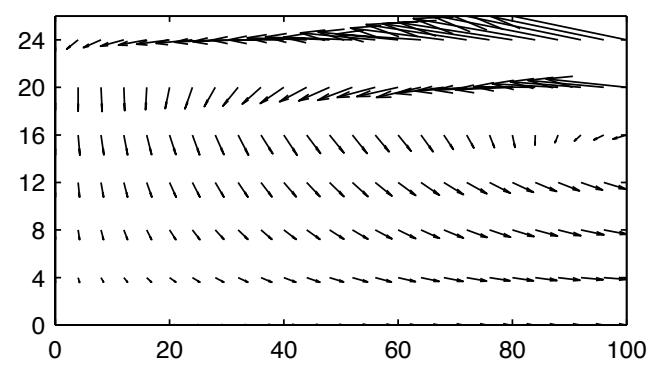

(d) at 3.95 years

Figure 6. Development of a recirculating flow cell in Case $A$ in the region of $[0,200 m] \times[0,22 m]$. 

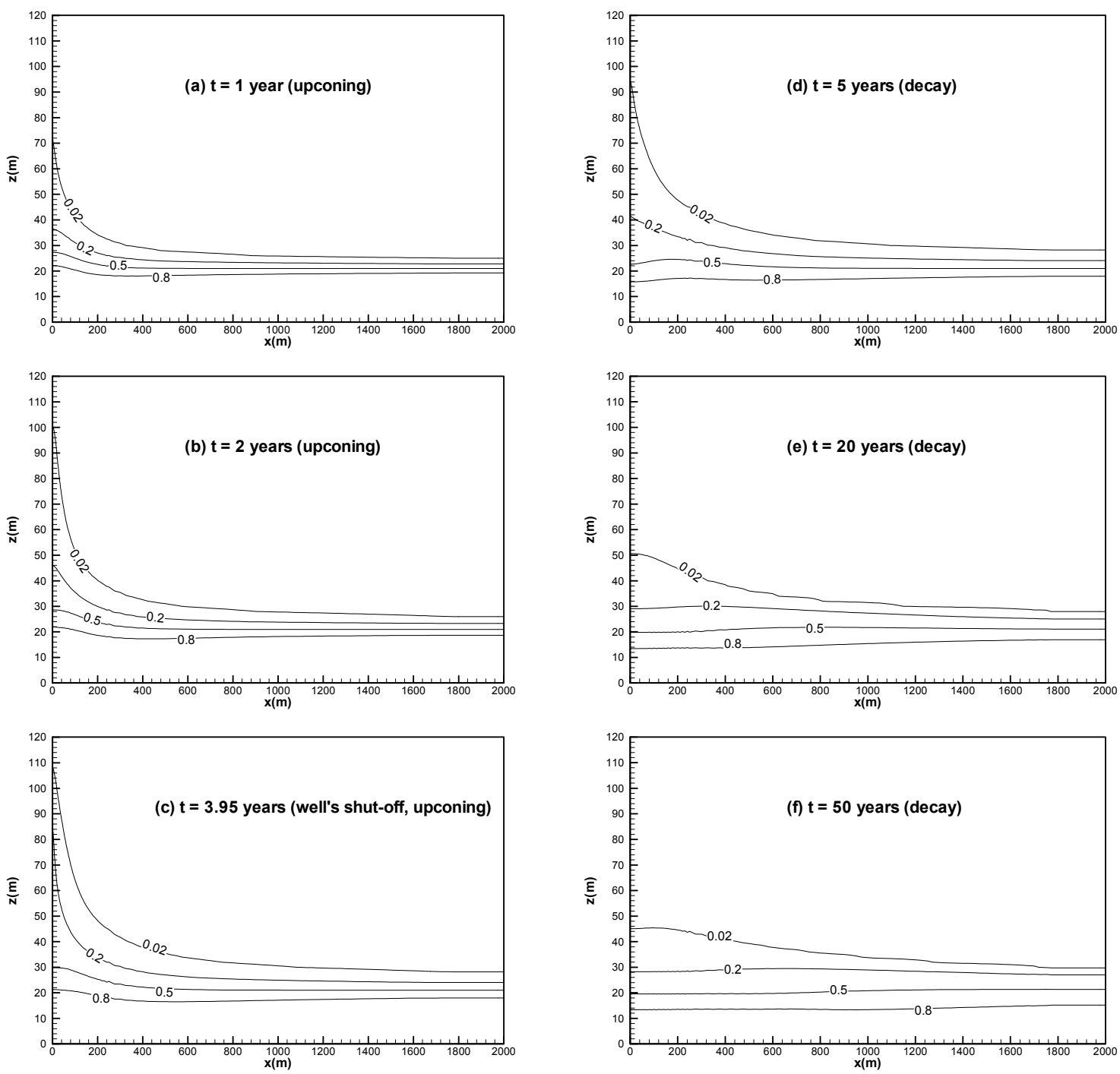

Figure 7. Transient distributions of normalized mass fraction in Case $\mathrm{A}\left(\alpha_{L}=1.0 \mathrm{~m}\right.$ and $\alpha_{T}=0.5 \mathrm{~m}$ ). The left-side panel indicates results under well pumpage, while the right-side one indicates results during the period of decay following the well's shutoff. 


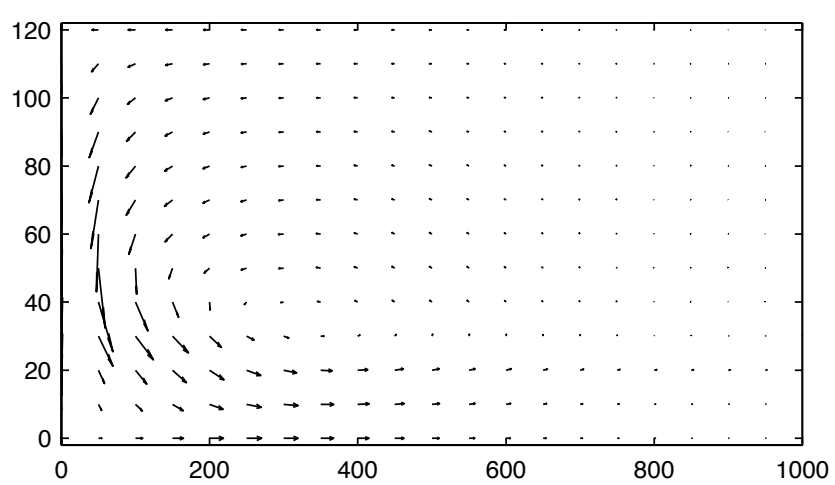

Figure 8. Velocity field at $t=5$ years during the period of decay following the well's shutoff for Case A. 

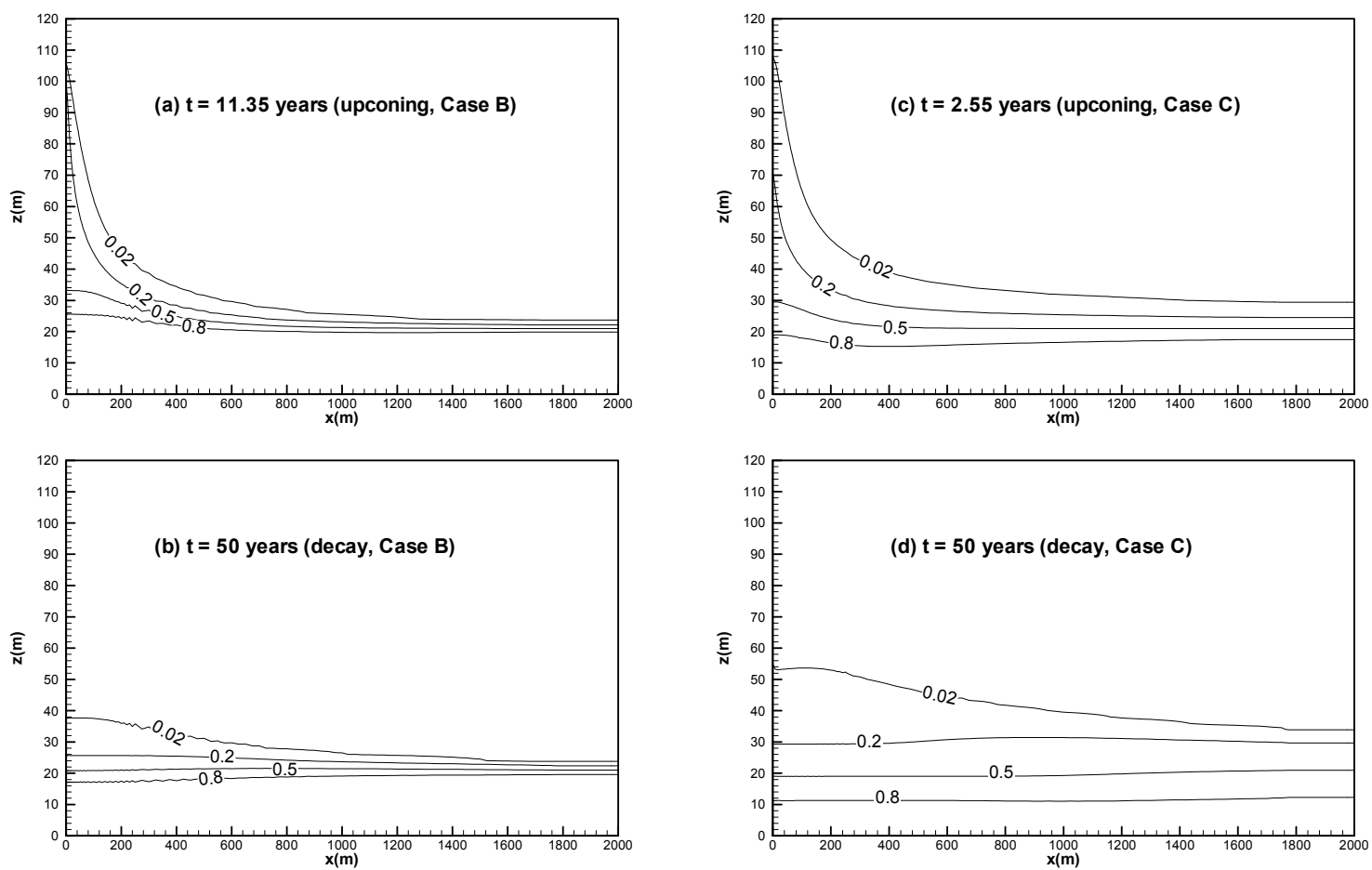

Figure 9. The normalized mass fraction distributions at (a) 11.35 years and (b) 50 years in Case B $\left(\alpha_{L}=0.2 \mathrm{~m}\right.$ and $\left.\alpha_{T}=0.02 \mathrm{~m}\right)$, and (c) at 2.55 years and (d) at 50 years in Case C $\left(\alpha_{L}=10 \mathrm{~m}\right.$ and $\left.\alpha_{T}=1.0 \mathrm{~m}\right)$. 

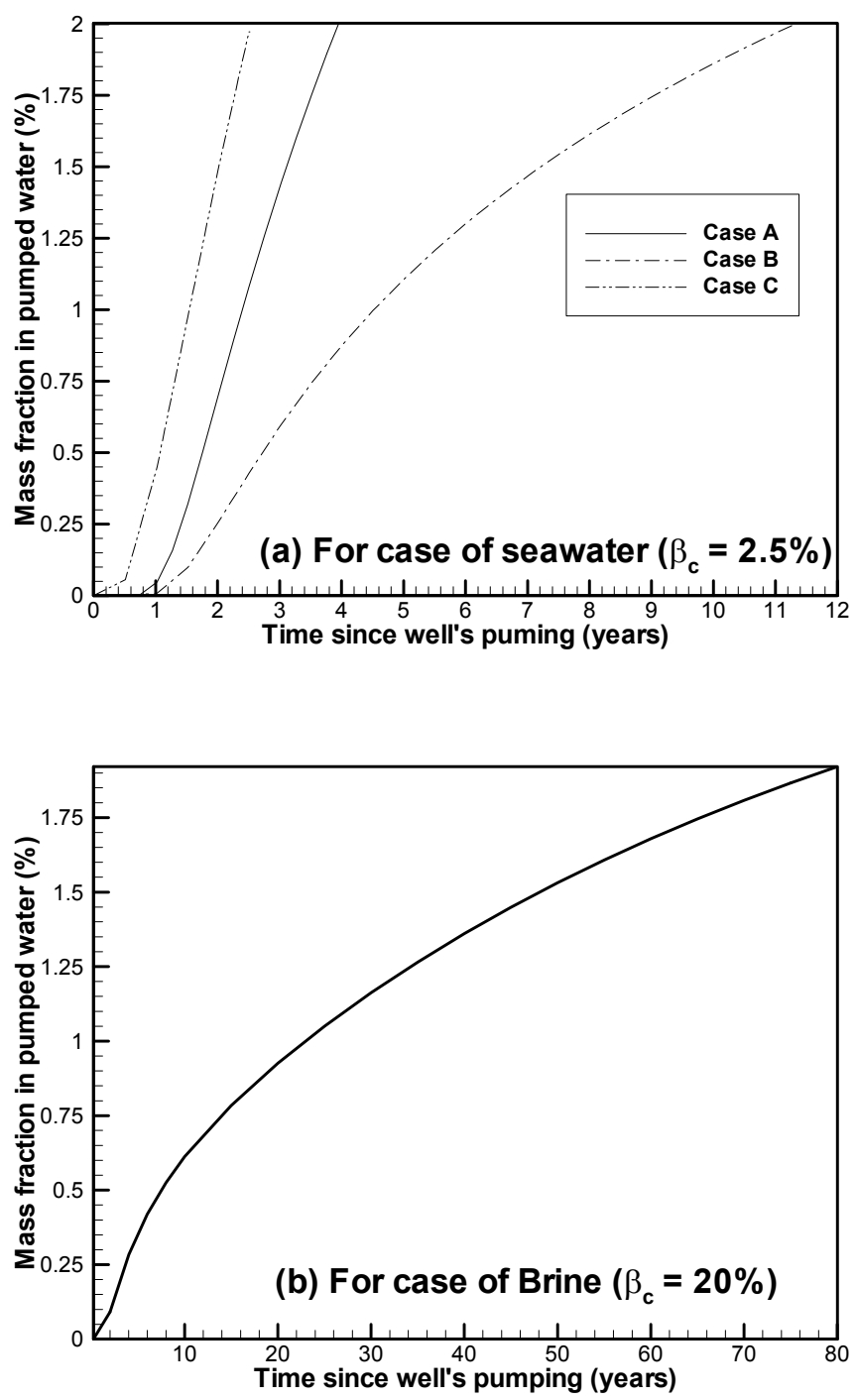

Figure 10. Breakthrough curves of the normalized mass fraction in the pumped water in the case of (a) seawater $\left(\beta_{c}=0.025\right)$ and (b) brine $\left(\beta_{c}=0.20\right)$. 

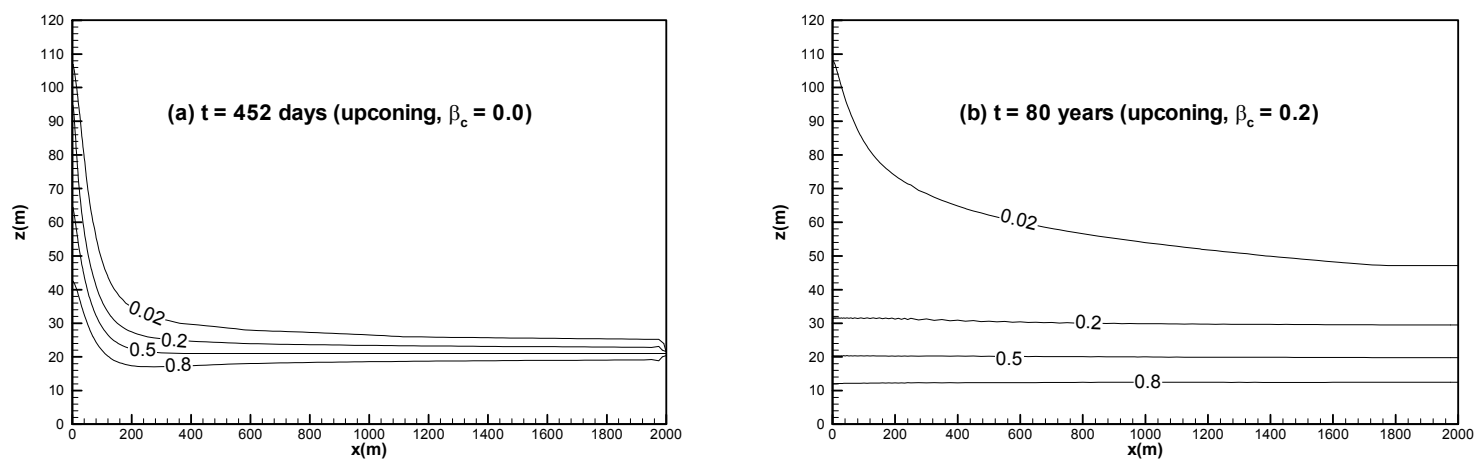

Figure 11. The normalized mass fraction distributions of (a) a tracer $\left(\beta_{c}=0.0\right)$ at 452 days of pumping, and (b) brine ( $\left.\beta_{c}=0.20\right)$ at 80 years of pumping in the case of $\alpha_{L}=1.0 \mathrm{~m}$ and $\alpha_{T}=0.5 m$. 